\title{
Some Existence Results for Impulsive Nonlinear Fractional Differential Equations with Closed Boundary Conditions
}

\author{
Hilmi Ergören ${ }^{1}$ and Adem Kiliçman ${ }^{2}$ \\ ${ }^{1}$ Department of Mathematics, Faculty of Sciences, Yuzuncu Yil University, 65080 Van, Turkey \\ ${ }^{2}$ Department of Mathematics and Institute for Mathematical Research, University Putra Malaysia, \\ 43400 Serdang, Malaysia \\ Correspondence should be addressed to Adem Kiliçman, akilicman@putra.upm.edu.my \\ Received 30 September 2012; Accepted 22 October 2012 \\ Academic Editor: Beata Rzepka
}

Copyright (c) 2012 H. Ergören and A. Kiliçman. This is an open access article distributed under the Creative Commons Attribution License, which permits unrestricted use, distribution, and reproduction in any medium, provided the original work is properly cited.

We investigate some existence results for the solutions to impulsive fractional differential equations having closed boundary conditions. Our results are based on contracting mapping principle and Burton-Kirk fixed point theorem.

\section{Introduction}

This paper considers the existence and uniqueness of the solutions to the closed boundary value problem (BVP), for the following impulsive fractional differential equation:

$$
\begin{gathered}
{ }^{C} D^{\alpha} x(t)=f(t, x(t)), \quad t \in J:=[0, T], t \neq t_{k}, 1<\alpha \leq 2, \\
\Delta x\left(t_{k}\right)=I_{k}\left(x\left(t_{k}^{-}\right)\right), \quad \Delta x^{\prime}\left(t_{k}\right)=I_{k}^{*}\left(x\left(t_{k}^{-}\right)\right), \quad k=1,2, \ldots, p, \\
x(T)=a x(0)+b T x^{\prime}(0), \quad T x^{\prime}(T)=c x(0)+d T x^{\prime}(0),
\end{gathered}
$$

where ${ }^{C} D^{\alpha}$ is Caputo fractional derivative, $f \in C(J \times R, R), I_{k}, I_{k}^{*} \in C(R, R)$,

$$
\Delta x\left(t_{k}\right)=x\left(t_{k}^{+}\right)-x\left(t_{k}^{-}\right),
$$


with

$$
x\left(t_{k}^{+}\right)=\lim _{h \rightarrow 0^{+}} x\left(t_{k}+h\right), \quad x\left(t_{k}^{-}\right)=\lim _{h \rightarrow 0^{-}} x\left(t_{k}+h\right),
$$

and $\Delta x^{\prime}\left(t_{k}\right)$ has a similar meaning for $x^{\prime}(t)$, where

$$
0=t_{0}<t_{1}<t_{2}<\cdots<t_{p}<t_{p+1}=T,
$$

$a, b, c$, and $d$ are real constants with $\Delta:=c(1-b)+(1-a)(1-d) \neq 0$.

The boundary value problems for nonlinear fractional differential equations have been addressed by several researchers during last decades. That is why, the fractional derivatives serve an excellent tool for the description of hereditary properties of various materials and processes. Actually, fractional differential equations arise in many engineering and scientific disciplines such as, physics, chemistry, biology, electrochemistry, electromagnetic, control theory, economics, signal and image processing, aerodynamics, and porous media (see [17]). For some recent development, see, for example, [8-14].

On the other hand, theory of impulsive differential equations for integer order has become important and found its extensive applications in mathematical modeling of phenomena and practical situations in both physical and social sciences in recent years. One can see a noticeable development in impulsive theory. For instance, for the general theory and applications of impulsive differential equations we refer the readers to [15-17].

Moreover, boundary value problems for impulsive fractional differential equations have been studied by some authors (see [18-20] and references therein). However, to the best of our knowledge, there is no study considering closed boundary value problems for impulsive fractional differential equations.

Here, we notice that the closed boundary conditions in (1.1) include quasi-periodic boundary conditions $(b=c=0)$ and interpolate between periodic $(a=d=1, b=c=0)$ and antiperiodic $(a=d=-1, b=c=0)$ boundary conditions.

Motivated by the mentioned recent work above, in this study, we investigate the existence and uniqueness of solutions to the closed boundary value problem for impulsive fractional differential equation (1.1). Throughout this paper, in Section 2, we present some notations and preliminary results about fractional calculus and differential equations to be used in the following sections. In Section 3, we discuss some existence and uniqueness results for solutions of BVP (1.1), that is, the first one is based on Banach's fixed point theorem, the second one is based on the Burton-Kirk fixed point theorem. At the end, we give an illustrative example for our results.

\section{Preliminaries}

Let us set $J_{0}=\left[0, t_{1}\right], J_{1}=\left(t_{1}, t_{2}\right], \ldots, J_{k-1}=\left(t_{k-1}, t_{k}\right], J_{k}=\left(t_{k}, t_{k+1}\right], J^{\prime}:=[0, T] \backslash\left\{t_{1}, t_{2}, \ldots, t_{p}\right\}$ and introduce the set of functions:

$P C(J, R)=\left\{x: J \rightarrow R: x \in C\left(\left(t_{k}, t_{k+1}\right], R\right), k=0,1,2, \ldots, p\right.$ and there exist $x\left(t_{k}^{+}\right)$and $x\left(t_{k}^{-}\right), k=1,2, \ldots, p$ with $\left.x\left(t_{k}^{-}\right)=x\left(t_{k}\right)\right\}$ and

$P C^{1}(J, R)=\left\{x \in P C(J, R), x^{\prime} \in C\left(\left(t_{k}, t_{k+1}\right], R\right), k=0,1,2, \ldots, p\right.$ and there exist $x^{\prime}\left(t_{k}^{+}\right)$ and $x^{\prime}\left(t_{k}^{-}\right), k=1,2, \ldots, p$ with $\left.x^{\prime}\left(t_{k}^{-}\right)=x^{\prime}\left(t_{k}\right)\right\}$ which is a Banach space with the norm $\|x\|=$ $\sup _{t \in J}\left\{\|x\|_{P C},\left\|x^{\prime}\right\|_{P C}\right\}$ where $\|x\|_{P C}:=\sup \{|x(t)|: t \in J\}$.

The following definitions and lemmas were given in [4]. 
Definition 2.1. The fractional (arbitrary) order integral of the function $h \in L^{1}\left(J, R_{+}\right)$of order $\alpha \in R_{+}$is defined by

$$
I_{0}^{\alpha} h(t)=\frac{1}{\Gamma(\alpha)} \int_{0}^{t}(t-s)^{\alpha-1} h(s) d s,
$$

where $\Gamma(\cdot)$ is the Euler gamma function.

Definition 2.2. For a function $h$ given on the interval $J$, Caputo fractional derivative of order $\alpha>0$ is defined by

$$
{ }^{C} D_{0^{+}}^{\alpha} h(t)=\frac{1}{\Gamma(n-\alpha)} \int_{0}^{t}(t-s)^{n-\alpha-1} h^{(n)}(s) d s, \quad n=[\alpha]+1,
$$

where the function $h(t)$ has absolutely continuous derivatives up to order $(n-1)$.

Lemma 2.3. Let $\alpha>0$, then the differential equation

$$
{ }^{C} D^{\alpha} h(t)=0
$$

has solutions

$$
h(t)=c_{0}+c_{1} t+c_{2} t^{2}+\cdots+c_{n-1} t^{n-1}, \quad c_{i} \in R, i=0,1,2, \ldots, n-1, n=[\alpha]+1 .
$$

The following lemma was given in $[4,10]$.

Lemma 2.4. Let $\alpha>0$, then

$$
I^{\alpha C} D^{\alpha} h(t)=h(t)+c_{0}+c_{1} t+c_{2} t^{2}+\cdots+c_{n-1} t^{n-1},
$$

for some $c_{i} \in R, i=0,1,2, \ldots, n-1, n=[\alpha]+1$. [21].

The following theorem is known as Burton-Kirk fixed point theorem and proved in

Theorem 2.5. Let $X$ be a Banach space and $A, D: X \rightarrow X$ two operators satisfying:

(a) $A$ is a contraction, and

(b) $D$ is completely continuous.

Then either

(i) the operator equation $x=A(x)+D(x)$ has a solution, or

(ii) the set $\varepsilon=\{x \in X: x=\lambda A(x / \lambda)+\lambda D(x)\}$ is unbounded for $\lambda \in(0,1)$.

Theorem 2.6 (see [22], Banach's fixed point theorem). Let $S$ be a nonempty closed subset of a Banach space $X$, then any contraction mapping $T$ of $S$ into itself has a unique fixed point. 
Next we prove the following lemma.

Lemma 2.7. Let $1<\alpha \leq 2$ and let $h: J \rightarrow R$ be continuous. A function $x(t)$ is a solution of the fractional integral equation:

$$
x(t)=\left\{\begin{array}{l}
\int_{0}^{t} \frac{(t-s)^{\alpha-1}}{\Gamma(\alpha)} h(s) d s+\Omega_{1}(t) \Lambda_{1}+\Omega_{2}(t) \Lambda_{2}, \quad t \in J_{0} \\
\int_{t_{k}}^{t} \frac{(t-s)^{\alpha-1}}{\Gamma(\alpha)} h(s) d s+\left[1+\Omega_{1}(t)\right] \sum_{i=1}^{k} \int_{t_{i-1}}^{t_{i}} \frac{\left(t_{i}-s\right)^{\alpha-1}}{\Gamma(\alpha)} h(s) d s \\
+\left[\left(T-t_{k}\right) \Omega_{1}(t)+\Omega_{2}(t)+\left(t-t_{k}\right)\right] \sum_{i=1}^{k} \int_{t_{i-1}}^{t_{i}} \frac{\left(t_{i}-s\right)^{\alpha-2}}{\Gamma(\alpha-1)} h(s) d s \\
+\left[1+\Omega_{1}(t)\right] \sum_{i=1}^{k-1}\left(t_{k}-t_{i}\right) \int_{t_{i-1}}^{t_{i}} \frac{\left(t_{i}-s\right)^{\alpha-2}}{\Gamma(\alpha-1)} h(s) d s+\Omega_{1}(t) \int_{t_{k}}^{T} \frac{(T-s)^{\alpha-1}}{\Gamma(\alpha)} h(s) d s \\
+\Omega_{2}(t) \int_{t_{k}}^{T} \frac{(T-s)^{\alpha-2}}{\Gamma(\alpha-1)} h(s) d s+\left[1+\Omega_{1}(t)\right]\left[\sum_{i=1}^{k} I_{i}\left(x\left(t_{i}^{-}\right)\right)+I_{k}^{*}\left(x\left(t_{k}^{-}\right)\right)\right] \\
+\Omega_{1}(t) \sum_{i=1}^{k-1}\left(T-t_{i}\right) I_{i}^{*}\left(x\left(t_{i}^{-}\right)\right)+\Omega_{2}(t) \sum_{i=1}^{k-1} I_{i}^{*}\left(x\left(t_{i}^{-}\right)\right) \\
+\sum_{i=1}^{k-1}\left(t-t_{i}\right) I_{i}^{*}\left(x\left(t_{i}^{-}\right)\right), \quad t \in J_{k}, k=1,2, \ldots, p
\end{array}\right.
$$

if and only if $x(t)$ is a solution of the fractional BVP

$$
\begin{gathered}
{ }^{C} D^{\alpha} x(t)=h(t), \quad t \in J^{\prime}, \\
\Delta x\left(t_{k}\right)=I_{k}\left(x\left(t_{k}^{-}\right)\right), \quad \Delta x^{\prime}\left(t_{k}\right)=I_{k}^{*}\left(x\left(t_{k}^{-}\right)\right), \\
x(T)=a x(0)+b T x^{\prime}(0), \quad T x^{\prime}(T)=c x(0)+d T x^{\prime}(0),
\end{gathered}
$$

where

$$
\begin{aligned}
\Lambda_{1}:= & \int_{t_{k}}^{T} \frac{(T-s)^{\alpha-1}}{\Gamma(\alpha)} h(s) d s+\sum_{i=1}^{k} \int_{t_{i-1}}^{t_{i}} \frac{\left(t_{i}-s\right)^{\alpha-1}}{\Gamma(\alpha)} h(s) d s \\
& +\sum_{i=1}^{k}\left(T-t_{k}\right) \int_{t_{i-1}}^{t_{i}} \frac{\left(t_{i}-s\right)^{\alpha-2}}{\Gamma(\alpha-1)} h(s) d s+\sum_{i=1}^{k-1}\left(t_{k}-t_{i}\right) \int_{t_{i-1}}^{t_{i}} \frac{\left(t_{i}-s\right)^{\alpha-2}}{\Gamma(\alpha-1)} h(s) d s \\
& +\sum_{i=1}^{k} I_{i}\left(x\left(t_{i}^{-}\right)\right)+\sum_{i=1}^{k-1}\left(T-t_{i}\right) I_{i}^{*}\left(x\left(t_{i}^{-}\right)\right)+I_{k}^{*}\left(x\left(t_{k}^{-}\right)\right),
\end{aligned}
$$




$$
\begin{gathered}
\Lambda_{2}:=\int_{t_{k}}^{T} \frac{(T-s)^{\alpha-2}}{\Gamma(\alpha-1)} h(s) d s+\sum_{i=1}^{k} \int_{t_{i-1}}^{t_{i}} \frac{\left(t_{i}-s\right)^{\alpha-2}}{\Gamma(\alpha-1)} h(s) d s+\sum_{i=1}^{k-1} I_{i}^{*}\left(x\left(t_{i}^{-}\right)\right), \\
\Omega_{1}(t):=-\frac{(1-d)}{\Delta}-\frac{c t}{T \Delta} \\
\Omega_{2}(t):=\frac{(1-b) T}{\Delta}-\frac{(1-a) t}{\Delta} .
\end{gathered}
$$

Proof. Let $x$ be the solution of (2.7). If $t \in J_{0}$, then Lemma 2.4 implies that

$$
\begin{gathered}
x(t)=I^{\alpha} h(t)-c_{0}-c_{1} t=\int_{0}^{t} \frac{(t-s)^{\alpha-1}}{\Gamma(\alpha)} h(s) d s-c_{0}-c_{1} t, \\
x^{\prime}(t)=\int_{0}^{t} \frac{(t-s)^{\alpha-2}}{\Gamma(\alpha-1)} h(s) d s-c_{1},
\end{gathered}
$$

for some $c_{0}, c_{1} \in R$.

If $t \in J_{1}$, then Lemma 2.4 implies that

$$
\begin{gathered}
x(t)=\int_{t_{1}}^{t} \frac{(t-s)^{\alpha-1}}{\Gamma(\alpha)} h(s) d s-d_{0}-d_{1}\left(t-t_{1}\right), \\
x^{\prime}(t)=\int_{t_{1}}^{t} \frac{(t-s)^{\alpha-2}}{\Gamma(\alpha-1)} h(s) d s-d_{1}
\end{gathered}
$$

for some $d_{0}, d_{1} \in R$. Thus we have

$$
\begin{gathered}
x\left(t_{1}^{-}\right)=\int_{t_{0}}^{t_{1}} \frac{\left(t_{1}-s\right)^{\alpha-1}}{\Gamma(\alpha)} h(s) d s-c_{0}-c_{1} t_{1}, \quad x\left(t_{1}^{+}\right)=-d_{0}, \\
x^{\prime}\left(t_{1}^{-}\right)=\int_{t_{0}}^{t_{1}} \frac{\left(t_{1}-s\right)^{\alpha-2}}{\Gamma(\alpha-1)} h(s) d s-c_{1}, \quad x^{\prime}\left(t_{1}^{+}\right)=-d_{1} .
\end{gathered}
$$

Observing that

$$
\Delta x\left(t_{1}\right)=x\left(t_{1}^{+}\right)-x\left(t_{1}^{-}\right)=I_{1}\left(x\left(t_{1}^{-}\right)\right), \quad \Delta x^{\prime}\left(t_{1}\right)=x^{\prime}\left(t_{1}^{+}\right)-x^{\prime}\left(t_{1}^{-}\right)=I_{1}^{*}\left(x\left(t_{1}^{-}\right)\right),
$$


then we have

$$
\begin{gathered}
-d_{0}=\int_{t_{0}}^{t_{1}} \frac{\left(t_{1}-s\right)^{\alpha-1}}{\Gamma(\alpha)} h(s) d s-c_{0}-c_{1} t_{1}+I_{1}\left(x\left(t_{1}^{-}\right)\right), \\
-d_{1}=\int_{t_{0}}^{t_{1}} \frac{\left(t_{1}-s\right)^{\alpha-2}}{\Gamma(\alpha-1)} h(s) d s-c_{1}+I_{1}^{*}\left(x\left(t_{1}^{-}\right)\right),
\end{gathered}
$$

hence, for $t \in\left(t_{1}, t_{2}\right]$,

$$
\begin{aligned}
x(t)= & \int_{t_{1}}^{t} \frac{(t-s)^{\alpha-1}}{\Gamma(\alpha)} h(s) d s+\int_{t_{0}}^{t_{1}} \frac{\left(t_{1}-s\right)^{\alpha-1}}{\Gamma(\alpha)} h(s) d s \\
& +\left(t-t_{1}\right) \int_{t_{0}}^{t_{1}} \frac{\left(t_{1}-s\right)^{\alpha-2}}{\Gamma(\alpha-1)} h(s) d s+I_{1}\left(x\left(t_{1}^{-}\right)\right)+\left(t-t_{1}\right) I_{1}^{*}\left(x\left(t_{1}^{-}\right)\right)-c_{0}-c_{1} t, \\
x^{\prime}(t)= & \int_{t_{1}}^{t} \frac{(t-s)^{\alpha-2}}{\Gamma(\alpha-1)} h(s) d s+\int_{t_{0}}^{t_{1}} \frac{\left(t_{1}-s\right)^{\alpha-2}}{\Gamma(\alpha-1)} h(s) d s+I_{1}^{*}\left(x\left(t_{1}^{-}\right)\right)-c_{1} .
\end{aligned}
$$

If $t \in J_{2}$, then Lemma 2.4 implies that

$$
\begin{gathered}
x(t)=\int_{t_{2}}^{t} \frac{(t-s)^{\alpha-1}}{\Gamma(\alpha)} h(s) d s-e_{0}-e_{1}\left(t-t_{2}\right), \\
x^{\prime}(t)=\int_{t_{2}}^{t} \frac{(t-s)^{\alpha-2}}{\Gamma(\alpha-1)} h(s) d s-e_{1}
\end{gathered}
$$

for some $e_{0}, e_{1} \in R$. Thus we have

$$
\begin{aligned}
& x\left(t_{2}^{-}\right)=\int_{t_{1}}^{t_{2}} \frac{\left(t_{2}-s\right)^{\alpha-1}}{\Gamma(\alpha)} h(s) d s+\int_{t_{0}}^{t_{1}} \frac{\left(t_{1}-s\right)^{\alpha-1}}{\Gamma(\alpha)} h(s) d s \\
& +\left(t_{2}-t_{1}\right) \int_{t_{0}}^{t_{1}} \frac{\left(t_{1}-s\right)^{\alpha-2}}{\Gamma(\alpha-1)} h(s) d s+I_{1}\left(x\left(t_{1}^{-}\right)\right)+\left(t_{2}-t_{1}\right) I_{1}^{*}\left(x\left(t_{1}^{-}\right)\right)-c_{0}-c_{1} t_{2}, \\
& x\left(t_{2}^{+}\right)=-e_{0}, \\
& x^{\prime}\left(t_{2}^{-}\right)=\int_{t_{1}}^{t_{2}} \frac{\left(t_{2}-s\right)^{\alpha-2}}{\Gamma(\alpha-1)} h(s) d s+\int_{t_{0}}^{t_{1}} \frac{\left(t_{1}-s\right)^{\alpha-2}}{\Gamma(\alpha-1)} h(s) d s+I_{1}^{*}\left(x\left(t_{1}^{-}\right)\right)-c_{1}, \\
& x^{\prime}\left(t_{2}^{+}\right)=-e_{1} \text {. }
\end{aligned}
$$

Similarly we observe that

$$
\Delta x\left(t_{2}\right)=x\left(t_{2}^{+}\right)-x\left(t_{2}^{-}\right)=I_{2}\left(x\left(t_{2}^{-}\right)\right), \quad \Delta x^{\prime}\left(t_{2}\right)=x^{\prime}\left(t_{2}^{+}\right)-x^{\prime}\left(t_{2}^{-}\right)=I_{2}^{*}\left(x\left(t_{2}^{-}\right)\right),
$$


thus we have

$$
\begin{aligned}
& -e_{0}=x\left(t_{2}^{-}\right)+I_{2}\left(x\left(t_{2}^{-}\right)\right), \\
& -e_{1}=x^{\prime}\left(t_{2}^{-}\right)+I_{2}^{*}\left(x\left(t_{2}^{-}\right)\right) .
\end{aligned}
$$

Hence, for $t \in\left(t_{2}, t_{3}\right]$,

$$
\begin{aligned}
x(t)= & \int_{t_{2}}^{t} \frac{(t-s)^{\alpha-1}}{\Gamma(\alpha)} h(s) d s+\int_{t_{0}}^{t_{1}} \frac{\left(t_{1}-s\right)^{\alpha-1}}{\Gamma(\alpha)} h(s) d s+\int_{t_{1}}^{t_{2}} \frac{\left(t_{2}-s\right)^{\alpha-1}}{\Gamma(\alpha)} h(s) d s \\
& +\left(t_{2}-t_{1}\right) \int_{t_{0}}^{t_{1}} \frac{\left(t_{1}-s\right)^{\alpha-2}}{\Gamma(\alpha-1)} h(s) d s \\
& +\left(t-t_{2}\right)\left[\int_{t_{0}}^{t_{1}} \frac{\left(t_{1}-s\right)^{\alpha-1}}{\Gamma(\alpha-1)} h(s) d s+\int_{t_{1}}^{t_{2}} \frac{\left(t_{2}-s\right)^{\alpha-1}}{\Gamma(\alpha-1)} h(s) d s\right] \\
& +I_{1}\left(x\left(t_{1}^{-}\right)\right)+I_{2}\left(x\left(t_{2}^{-}\right)\right)+\left(t-t_{1}\right) I_{1}^{*}\left(x\left(t_{1}^{-}\right)\right)+I_{2}^{*}\left(x\left(t_{2}^{-}\right)\right)-c_{0}-c_{1} t .
\end{aligned}
$$

By a similar process, if $t \in J_{k}$, then again from Lemma 2.4 we get

$$
\begin{aligned}
& x(t)=\left\{\begin{array}{l}
\int_{t_{k}}^{t} \frac{(t-s)^{\alpha-1}}{\Gamma(\alpha)} h(s) d s+\sum_{i=1}^{k} \int_{t_{i-1}}^{t_{i}} \frac{\left(t_{i}-s\right)^{\alpha-1}}{\Gamma(\alpha)} h(s) d s \\
\quad+\sum_{i=1}^{k}\left(t-t_{k}\right) \int_{t_{i-1}}^{t_{i}} \frac{\left(t_{i}-s\right)^{\alpha-2}}{\Gamma(\alpha-1)} h(s) d s+\sum_{i=1}^{k-1}\left(t_{k}-t_{i}\right) \int_{t_{i-1}}^{t_{i}} \frac{\left(t_{i}-s\right)^{\alpha-2}}{\Gamma(\alpha-1)} h(s) d s \\
\quad+\sum_{i=1}^{k} I_{i}\left(x\left(t_{i}^{-}\right)\right)+\sum_{i=1}^{k-1}\left(t-t_{i}\right) I_{i}^{*}\left(x\left(t_{i}^{-}\right)\right)+I_{k}^{*}\left(x\left(t_{k}^{-}\right)\right)-c_{0}-c_{1} t,
\end{array}\right. \\
& x^{\prime}(t)=\left\{\int_{t_{k}}^{t} \frac{(t-s)^{\alpha-2}}{\Gamma(\alpha-1)} h(s) d s+\sum_{i=1}^{k} \int_{t_{i-1}}^{t_{i}} \frac{\left(t_{i}-s\right)^{\alpha-2}}{\Gamma(\alpha-1)} h(s) d s+\sum_{i=1}^{k-1} I_{i}^{*}\left(x\left(t_{i}^{-}\right)\right)-c_{1} .\right.
\end{aligned}
$$

Now if we apply the conditions:

$$
x(T)=a x(0)+b T x^{\prime}(0), \quad T x^{\prime}(T)=c x(0)+d T x^{\prime}(0),
$$

we have

$$
\begin{gathered}
\Lambda_{1}=(1-a) c_{0}+T(1-b) c_{1}, \\
\Lambda_{2}=-\frac{c}{T} c_{0}+(1-d) c_{1}, \\
-c_{0}=-\frac{(1-d) \Lambda_{1}}{\Delta}+\frac{(1-b) T \Lambda_{2}}{\Delta}, \\
-c_{1}=-\frac{c \Lambda_{1}}{T \Delta}-\frac{(1-a) \Lambda_{2}}{\Delta} .
\end{gathered}
$$


In view of the relations (2.8), when the values of $-c_{0}$ and $-c_{1}$ are replaced in (2.9) and (2.20), the integral equation (2.7) is obtained.

Conversely, assume that $x$ satisfies the impulsive fractional integral equation (2.6), then by direct computation, it can be seen that the solution given by (2.6) satisfies (2.7). The proof is complete.

\section{Main Results}

Definition 3.1. A function $x \in P C^{1}(J, R)$ with its $\alpha$-derivative existing on $J^{\prime}$ is said to be a solution of (1.1), if $x$ satisfies the equation ${ }^{C} D^{\alpha} x(t)=f(t, x(t))$ on $J^{\prime}$ and satisfies the conditions:

$$
\begin{gathered}
\Delta x\left(t_{k}\right)=I_{k}\left(x\left(t_{k}^{-}\right)\right), \quad \Delta x^{\prime}\left(t_{k}\right)=I_{k}^{*}\left(x\left(t_{k}^{-}\right)\right), \\
x(T)=a x(0)+b T x^{\prime}(0), \quad T x^{\prime}(T)=c x(0)+d T x^{\prime}(0) .
\end{gathered}
$$

For the sake of convenience, we define

$$
\Omega_{1}^{*}=\sup _{t \in J}\left|\Omega_{1}(t)\right|, \quad \Omega_{2}^{*}=\sup _{t \in J}\left|\Omega_{2}(t)\right|, \quad \Omega_{1}^{* *}=\sup _{t \in J}\left|\Omega_{1}^{\prime}(t)\right|, \quad \Omega_{2}^{* *}=\sup _{t \in J}\left|\Omega_{2}^{\prime}(t)\right| .
$$

The followings are main results of this paper.

Theorem 3.2. Assume that

(A1) the function $f: J \times R \rightarrow R$ is continuous and there exists a constant $L_{1}>0$ such that $\|f(t, u)-f(t, v)\| \leq L_{1}\|u-v\|$, for all $t \in J$, and $u, v \in R$,

(A2) $I_{k}, I_{k}^{*}: R \rightarrow R$ are continuous, and there exist constants $L_{2}>0$ and $L_{3}>0$ such that $\left\|I_{k}(u)-I_{k}(v)\right\| \leq L_{2}\|u-v\|,\left\|I_{k}^{*}(u)-I_{k}^{*}(v)\right\| \leq L_{3}\|u-v\|$ for each $u, v \in R$ and $k=1,2, \ldots, p$.

Moreover, consider the following:

$$
\begin{gathered}
{\left[\frac{L_{1} T^{\alpha}}{\Gamma(\alpha+1)}\left(1+\Omega_{1}^{*}\right)(1+p+2 p \alpha)+\frac{L_{1} T^{\alpha-1}}{\Gamma(\alpha)} \Omega_{2}^{*}(1+p)\right.} \\
\left.+\left(1+\Omega_{1}^{*}\right)\left(p L_{2}+L_{3}\right)+\left(\Omega_{1}^{*} T+\Omega_{2}^{*}+T\right) p L_{3}\right]<1 .
\end{gathered}
$$

Then, BVP (1.1) has a unique solution on $J$.

Proof. Define an operator $F: P C^{1}(J, R) \rightarrow P C^{1}(J, R)$ by

$$
\begin{aligned}
(F x)(t)= & \int_{t_{k}}^{t} \frac{(t-s)^{\alpha-1}}{\Gamma(\alpha)} f(s, x(s)) d s+\left[1+\Omega_{1}(t)\right] \sum_{i=1}^{k} \int_{t_{i-1}}^{t_{i}} \frac{\left(t_{i}-s\right)^{\alpha-1}}{\Gamma(\alpha)} f(s, x(s)) d s \\
& +\left[\left(T-t_{k}\right) \Omega_{1}(t)+\Omega_{2}(t)+\left(t-t_{k}\right)\right] \sum_{i=1}^{k} \int_{t_{i-1}}^{t_{i}} \frac{\left(t_{i}-s\right)^{\alpha-2}}{\Gamma(\alpha-1)} f(s, x(s)) d s
\end{aligned}
$$


Abstract and Applied Analysis

$$
\begin{aligned}
& +\left[1+\Omega_{1}(t)\right] \sum_{i=1}^{k-1}\left(t_{k}-t_{i}\right) \int_{t_{i-1}}^{t_{i}} \frac{\left(t_{i}-s\right)^{\alpha-2}}{\Gamma(\alpha-1)} f(s, x(s)) d s \\
& +\Omega_{1}(t) \int_{t_{k}}^{T} \frac{(T-s)^{\alpha-1}}{\Gamma(\alpha)} f(s, x(s)) d s \\
& +\Omega_{2}(t) \int_{t_{k}}^{T} \frac{(T-s)^{\alpha-2}}{\Gamma(\alpha-1)} f(s, x(s)) d s+\left[1+\Omega_{1}(t)\right]\left[\sum_{i=1}^{k} I_{i}\left(x\left(t_{i}^{-}\right)\right)+I_{k}^{*}\left(x\left(t_{k}^{-}\right)\right)\right] \\
& +\Omega_{1}(t) \sum_{i=1}^{k-1}\left(T-t_{i}\right) I_{i}^{*}\left(x\left(t_{i}^{-}\right)\right)+\Omega_{2}(t) \sum_{i=1}^{k-1} I_{i}^{*}\left(x\left(t_{i}^{-}\right)\right)+\sum_{i=1}^{k-1}\left(t-t_{i}\right) I_{i}^{*}\left(x\left(t_{i}^{-}\right)\right) .
\end{aligned}
$$

Now, for $x, y \in P C(J, R)$ and for each $t \in J$, we obtain

$$
\begin{aligned}
|(F x)(t)-(F y)(t)| \leq & \int_{t_{k}}^{t} \frac{(t-s)^{\alpha-1}}{\Gamma(\alpha)}|f(s, x(s))-f(s, y(s))| d s \\
& +\left|1+\Omega_{1}(t)\right| \sum_{i=1}^{k} \int_{t_{i-1}}^{t_{i}} \frac{\left(t_{i}-s\right)^{\alpha-1}}{\Gamma(\alpha)}|f(s, x(s))-f(s, y(s))| d s \\
& +\left|\left(T-t_{k}\right) \Omega_{1}(t)+\Omega_{2}(t)+\left(t-t_{k}\right)\right| \\
& +\sum_{i=1}^{k} \int_{t_{i-1}}^{t_{i}} \frac{\left(t_{i}-s\right)^{\alpha-2}}{\Gamma(\alpha-1)}|f(s, x(s))-f(s, y(s))| d s \\
& +\left|1+\Omega_{1}(t)\right| \sum_{i=1}^{k-1}\left(t_{k}-t_{i}\right) \int_{t_{i-1}}^{t_{i}} \frac{\left(t_{i}-s\right)^{\alpha-2}}{\Gamma(\alpha-1)}|f(s, x(s))-f(s, y(s))| d s \\
& +\left|\Omega_{1}(t)\right| \int_{t_{k}}^{T} \frac{(T-s)^{\alpha-1}}{\Gamma(\alpha)}|f(s, x(s))-f(s, y(s))| d s \\
& +\left|\Omega_{2}(t)\right| \int_{t_{k}}^{T} \frac{(T-s)^{\alpha-2}}{\Gamma(\alpha-1)}|f(s, x(s))-f(s, y(s))| d s \\
& +\left|1+\Omega_{1}(t)\right|\left[\sum_{i=1}^{k}\left|I_{i}\left(x\left(t_{i}^{-}\right)\right)-I_{i}\left(y\left(t_{i}^{-}\right)\right)\right|+\left|I_{k}^{*}\left(x\left(t_{k}^{-}\right)\right)-I_{k}^{*}\left(y\left(t_{k}^{-}\right)\right)\right|\right] \\
& +\left|\Omega_{1}(t)\right| \sum_{i=1}^{k-1}\left(T-t_{i}\right)\left|I_{i}^{*}\left(x\left(t_{i}^{-}\right)\right)-I_{i}^{*}\left(y\left(t_{i}^{-}\right)\right)\right| \\
& +\left|\Omega_{2}(t)\right| \sum_{i=1}^{k-1}\left|I_{i}^{*}\left(x\left(t_{i}^{-}\right)\right)-I_{i}^{*}\left(y\left(t_{i}^{-}\right)\right)\right| \\
& +\sum_{i=1}^{k-1}\left|t-t_{i}\right|\left|I_{i}^{*}\left(x\left(t_{i}^{-}\right)\right)-I_{i}^{*}\left(y\left(t_{i}^{-}\right)\right)\right|, \\
& \\
&
\end{aligned}
$$




$$
\begin{aligned}
\|(F x)(t)-(F y)(t)\| \leq & {\left[\frac{L_{1} T^{\alpha}}{\Gamma(\alpha+1)}\left(1+\Omega_{1}^{*}\right)(1+p+2 p \alpha)+\frac{L_{1} T^{\alpha-1}}{\Gamma(\alpha)} \Omega_{2}^{*}(1+p)\right.} \\
& \left.+\left(1+\Omega_{1}^{*}\right)\left(p L_{2}+L_{3}\right)+\left(\Omega_{1}^{*} T+\Omega_{2}^{*}+T\right) p L_{3}\right]\|x(s)-y(s)\| .
\end{aligned}
$$

Therefore, by (3.3), the operator $F$ is a contraction mapping. In a consequence of Banach's fixed theorem, the BVP (1.1) has a unique solution. Now, our second result relies on the Burton-Kirk fixed point theorem.

Theorem 3.3. Assume that (A1)-(A2) hold, and

(A3) there exist constants $M_{1}>0, M_{2}>0, M_{3}>0$ such that $\|f(t, u)\| \leq M_{1},\left\|I_{k}(u)\right\| \leq M_{2}$, $\left\|I_{k}^{*}(u)\right\| \leq M_{3}$ for each $u, v \in R$ and $k=1,2, \ldots, p$.

Then the BVP (1.1) has at least one solution on J.

Proof. We define the operators $A, D: P C^{1}(J, R) \rightarrow P C^{1}(J, R)$ by

$$
\begin{aligned}
(A x)(t)= & {\left[1+\Omega_{1}(t)\right]\left[\sum_{i=1}^{k} I_{i}\left(x\left(t_{i}^{-}\right)\right)+I_{k}^{*}\left(x\left(t_{k}^{-}\right)\right)\right] } \\
& +\Omega_{1}(t) \sum_{i=1}^{k-1}\left(T-t_{i}\right) I_{i}^{*}\left(x\left(t_{i}^{-}\right)\right)+\Omega_{2}(t) \sum_{i=1}^{k-1} I_{i}^{*}\left(x\left(t_{i}^{-}\right)\right)+\sum_{i=1}^{k-1}\left(t-t_{i}\right) I_{i}^{*}\left(x\left(t_{i}^{-}\right)\right), \\
(D x)(t)= & \int_{t_{k}}^{t} \frac{(t-s)^{\alpha-1}}{\Gamma(\alpha)} f(s, x(s)) d s+\left[1+\Omega_{1}(t)\right] \sum_{i=1}^{k} \int_{t_{i-1}}^{t_{i}} \frac{\left(t_{i}-s\right)^{\alpha-1}}{\Gamma(\alpha)} f(s, x(s)) d s \\
& +\left[\left(T-t_{k}\right) \Omega_{1}(t)+\Omega_{2}(t)+\left(t-t_{k}\right)\right] \sum_{i=1}^{k} \int_{t_{i-1}}^{t_{i}} \frac{\left(t_{i}-s\right)^{\alpha-2}}{\Gamma(\alpha-1)} f(s, x(s)) d s \\
& +\left[1+\Omega_{1}(t)\right] \sum_{i=1}^{k-1}\left(t_{k}-t_{i}\right) \int_{t_{i-1}}^{t_{i}} \frac{\left(t_{i}-s\right)^{\alpha-2}}{\Gamma(\alpha-1)} f(s, x(s)) d s \\
& +\Omega_{1}(t) \int_{t_{k}}^{T} \frac{(T-s)^{\alpha-1}}{\Gamma(\alpha)} f(s, x(s)) d s+\Omega_{2}(t) \int_{t_{k}}^{T} \frac{(T-s)^{\alpha-2}}{\Gamma(\alpha-1)} f(s, x(s)) d s .
\end{aligned}
$$

It is obvious that $A$ is contraction mapping for

$$
\left(1+\Omega_{1}^{*}\right)\left(p L_{2}+L_{3}\right)+\left(\Omega_{1}^{*} T+\Omega_{2}^{*}+T\right) p L_{3}<1
$$

Now, in order to check that $D$ is completely continuous, let us follow the sequence of the following steps. 
Step 1 (D is continuous). Let $\left\{x_{n}\right\}$ be a sequence such that $x_{n} \rightarrow x$ in $P C(J, R)$. Then for $t \in J$, we have

$$
\begin{aligned}
\left|\left(D x_{n}\right)(t)-(D x)(t)\right| \leq & \int_{t_{k}}^{t} \frac{(t-s)^{\alpha-1}}{\Gamma(\alpha)}\left|f\left(s, x_{n}(s)\right)-f(s, x(s))\right| d s \\
& +\left|1+\Omega_{1}(t)\right| \sum_{i=1}^{k} \int_{t_{i-1}}^{t_{i}} \frac{\left(t_{i}-s\right)^{\alpha-1}}{\Gamma(\alpha)}\left|f\left(s, x_{n}(s)\right)-f(s, x(s))\right| d s \\
& +\left|\left(T-t_{k}\right) \Omega_{1}(t)+\Omega_{2}(t)+\left(t-t_{k}\right)\right| \\
& \times \sum_{i=1}^{k} \int_{t_{i-1}}^{t_{i}} \frac{\left(t_{i}-s\right)^{\alpha-2}}{\Gamma(\alpha-1)}\left|f\left(s, x_{n}(s)\right)-f(s, x(s))\right| d s \\
& +\left|1+\Omega_{1}(t)\right| \sum_{i=1}^{k-1}\left(t_{k}-t_{i}\right) \int_{t_{i-1}}^{t_{i}} \frac{\left(t_{i}-s\right)^{\alpha-2}}{\Gamma(\alpha-1)}\left|f\left(s, x_{n}(s)\right)-f(s, x(s))\right| d s \\
& +\left|\Omega_{1}(t)\right| \int_{t_{k}}^{T} \frac{(T-s)^{\alpha-1}}{\Gamma(\alpha)}\left|f\left(s, x_{n}(s)\right)-f(s, x(s))\right| d s \\
& +\left|\Omega_{2}(t)\right| \int_{t_{k}}^{T} \frac{(T-s)^{\alpha-2}}{\Gamma(\alpha-1)}\left|f\left(s, x_{n}(s)\right)-f(s, x(s))\right| d s .
\end{aligned}
$$

Since $f$ is continuous function, we get

$$
\left\|\left(D x_{n}\right)(t)-(D x)(t)\right\| \longrightarrow 0 \quad \text { as } n \longrightarrow \infty
$$

Step 2 ( $D$ maps bounded sets into bounded sets in $P C(J, R)$ ). Indeed, it is enough to show that for any $r>0$, there exists a positive constant $l$ such that for each $x \in B_{r}=\{x \in P C(J, R)$ : $\|x\| \leq r\}$, we have $\|D(x)\| \leq l$. By (A3), we have for each $t \in J$,

$$
\begin{aligned}
|(D x)(t)| \leq & \int_{t_{k}}^{t} \frac{(t-s)^{\alpha-1}}{\Gamma(\alpha)}|f(s, x(s))| d s \\
& +\left|1+\Omega_{1}(t)\right| \sum_{i=1}^{k} \int_{t_{i-1}}^{t_{i}} \frac{\left(t_{i}-s\right)^{\alpha-1}}{\Gamma(\alpha)}|f(s, x(s))| d s \\
& +\left|\left(T-t_{k}\right) \Omega_{1}(t)+\Omega_{2}(t)+\left(t-t_{k}\right)\right| \sum_{i=1}^{k} \int_{t_{i-1}}^{t_{i}} \frac{\left(t_{i}-s\right)^{\alpha-2}}{\Gamma(\alpha-1)}|f(s, x(s))| d s \\
& +\left|1+\Omega_{1}(t)\right| \sum_{i=1}^{k-1}\left(t_{k}-t_{i}\right) \int_{t_{i-1}}^{t_{i}} \frac{\left(t_{i}-s\right)^{\alpha-2}}{\Gamma(\alpha-1)}|f(s, x(s))| d s
\end{aligned}
$$




$$
\begin{gathered}
+\left|\Omega_{1}(t)\right| \int_{t_{k}}^{T} \frac{(T-s)^{\alpha-1}}{\Gamma(\alpha)}|f(s, x(s))| d s+\left|\Omega_{2}(t)\right| \int_{t_{k}}^{T} \frac{(T-s)^{\alpha-2}}{\Gamma(\alpha-1)}|f(s, x(s))| d s, \\
\|D(x)\| \leq \frac{M_{1} T^{\alpha}}{\Gamma(\alpha+1)}\left(1+\Omega_{1}^{*}\right)(1+p+2 p \alpha)+\frac{M_{1} T^{\alpha-1}}{\Gamma(\alpha)} \Omega_{2}^{*}(1+p):=l .
\end{gathered}
$$

Step 3 ( $D$ maps bounded sets into equicontinuous sets in $P C^{1}(J, R)$ ). Let $\tau_{1}, \tau_{2} \in J_{k}, 0 \leq k \leq p$ with $\tau_{1}<\tau_{2}$ and let $B_{r}$ be a bounded set of $P C^{1}(J, R)$ as in Step 2, and let $x \in B_{r}$. Then

$$
\left|(D y)\left(\tau_{2}\right)-(D)\left(\tau_{1}\right)\right| \leq \int_{\tau_{1}}^{\tau_{2}}\left|(D y)^{\prime}(s)\right| d s \leq L\left(\tau_{2}-\tau_{1}\right),
$$

where

$$
\begin{aligned}
\left|(D x)^{\prime}(t)\right| \leq & \int_{t_{k}}^{t} \frac{(t-s)^{\alpha-2}}{\Gamma(\alpha-1)}|f(s, x(s))| d s+\left|\Omega_{1}^{\prime}(t)\right| \sum_{i=1}^{k} \int_{t_{i-1}}^{t_{i}} \frac{\left(t_{i}-s\right)^{\alpha-1}}{\Gamma(\alpha)}|f(s, x(s))| d s \\
& +\left|\left(T-t_{k}\right) \Omega_{1}^{\prime}(t)+\Omega_{2}^{\prime}(t)+1\right| \sum_{i=1}^{k} \int_{t_{i-1}}^{t_{i}} \frac{\left(t_{i}-s\right)^{\alpha-2}}{\Gamma(\alpha-1)}|f(s, x(s))| d s \\
& +\left|\Omega_{1}^{\prime}(t)\right| \sum_{i=1}^{k-1}\left(t_{k}-t_{i}\right) \int_{t_{i-1}}^{t_{i}} \frac{\left(t_{i}-s\right)^{\alpha-2}}{\Gamma(\alpha-1)}|f(s, x(s))| d s \\
& +\left|\Omega_{1}^{\prime}(t)\right| \int_{t_{k}}^{T} \frac{(T-s)^{\alpha-1}}{\Gamma(\alpha)}|f(s, x(s))| d s+\left|\Omega_{2}^{\prime}(t)\right| \int_{t_{k}}^{T} \frac{(T-s)^{\alpha-2}}{\Gamma(\alpha-1)}|f(s, x(s))| d s, \\
\leq & \frac{M_{1} T^{\alpha}}{\Gamma(\alpha+1)} \Omega_{1}^{*}(1+p+2 p \alpha)+\frac{M_{1} T^{\alpha-1}}{\Gamma(\alpha)}\left(1+\Omega_{2}^{*}\right)(1+p):=L .
\end{aligned}
$$

This implies that $A$ is equicontinuous on all the subintervals $J_{k}, k=0,1,2, \ldots, p$. Therefore, by the Arzela-Ascoli Theorem, the operator $D: P C^{1}(J, R) \rightarrow P C^{1}(J, R)$ is completely continuous.

To conclude the existence of a fixed point of the operator $A+D$, it remains to show that the set

$$
\varepsilon=\left\{x \in X: x=\lambda A\left(\frac{x}{\lambda}\right)+\lambda D(x) \text { for some } \lambda \in(0,1)\right\}
$$

is bounded.

Let $x \in \varepsilon$, for each $t \in J$,

$$
x(t)=\lambda D(x)(t)+\lambda A\left(\frac{x}{\lambda}\right)(t) .
$$


Hence, from (A3), we have

$$
\begin{aligned}
|x(t)| \leq & \lambda \int_{t_{k}}^{t} \frac{(t-s)^{\alpha-1}}{\Gamma(\alpha)}|f(s, x(s))| d s+\lambda\left|1+\Omega_{1}(t)\right| \sum_{i=1}^{k} \int_{t_{i-1}}^{t_{i}} \frac{\left(t_{i}-s\right)^{\alpha-1}}{\Gamma(\alpha)}|f(s, x(s))| d s \\
& +\lambda\left|\left(T-t_{k}\right) \Omega_{1}(t)+\Omega_{2}(t)+\left(t-t_{k}\right)\right| \sum_{i=1}^{k} \int_{t_{i-1}}^{t_{i}} \frac{\left(t_{i}-s\right)^{\alpha-2}}{\Gamma(\alpha-1)}|f(s, x(s))| d s \\
& +\lambda\left|1+\Omega_{1}(t)\right| \sum_{i=1}^{k-1}\left(t_{k}-t_{i}\right) \int_{t_{i-1}}^{t_{i}} \frac{\left(t_{i}-s\right)^{\alpha-2}}{\Gamma(\alpha-1)}|f(s, x(s))| d s \\
& +\lambda\left|\Omega_{1}(t)\right| \int_{t_{k}}^{T} \frac{(T-s)^{\alpha-1}}{\Gamma(\alpha)}|f(s, x(s))| d s+\lambda\left|\Omega_{2}(t)\right| \int_{t_{k}}^{T} \frac{(T-s)^{\alpha-2}}{\Gamma(\alpha-1)}|f(s, x(s))| d s \\
& +\lambda\left|1+\Omega_{1}(t)\right|\left[\sum_{i=1}^{k}\left|I_{i}\left(\frac{x\left(t_{i}^{-}\right)}{\lambda}\right)\right|+\left|I_{k}^{*}\left(\frac{x\left(t_{k}^{-}\right)}{\lambda}\right)\right|\right] \\
& +\lambda\left|\Omega_{1}(t)\right| \sum_{i=1}^{k-1}\left(T-t_{i}\right)\left|I_{i}^{*}\left(\frac{x\left(t_{i}^{-}\right)}{\lambda}\right)\right| \\
& +\lambda\left|\Omega_{2}(t)\right| \sum_{i=1}^{k-1}\left|I_{i}^{*}\left(\frac{x\left(t_{i}^{-}\right)}{\lambda}\right)\right|+\lambda \sum_{i=1}^{k-1}\left(t-t_{i}\right)\left|I_{i}^{*}\left(\frac{x\left(t_{i}^{-}\right)}{\lambda}\right)\right| \\
\|x(t)\| \leq & {\left[\frac{M_{1} T^{\alpha}}{\Gamma(\alpha+1)}\left(1+\Omega_{1}^{*}\right)(1+p+2 p \alpha)+\frac{M_{1} T^{\alpha-1}}{\Gamma(\alpha)} \Omega_{2}^{*}(1+p)\right.} \\
& \left.+\left(1+\Omega_{1}^{*}\right)\left(p M_{2}+M_{3}\right)+\left(\Omega_{1}^{*} T+\Omega_{2}^{*}+T\right) p M_{3}\right] .
\end{aligned}
$$

Consequently, we conclude the result of our theorem based on the Burton-Kirk fixed point theorem.

\section{An Example}

Consider the following impulsive fractional boundary value problem:

$$
\begin{gathered}
{ }^{C} D^{3 / 2} x(t)=\frac{\sin 2 t|x(t)|}{(t+5)^{2}(1+|x(t)|)}, \quad t \in[0,1], t \neq \frac{1}{3^{\prime}} \\
\Delta x\left(\frac{1}{3}\right)=\frac{\left|x\left(1^{-} / 3\right)\right|}{15+\left|x\left(1^{-} / 3\right)\right|}, \quad \Delta x^{\prime}\left(\frac{1}{3}\right)=\frac{\left|x^{\prime}\left(1^{-} / 3\right)\right|}{10+\left|x^{\prime}\left(1^{-} / 3\right)\right|^{\prime}} \\
x(1)=5 x(0)-2 x^{\prime}(0), \quad x^{\prime}(1)=x(0)+4 x^{\prime}(0) .
\end{gathered}
$$


Here, $a=5, b=-2, c=1, d=4, \alpha=3 / 2, T=1, p=1$. Obviously, $L_{1}=1 / 25, L_{2}=1 / 15$, $L_{3}=1 / 10, \Omega_{1}^{*}=4 / 15, \Omega_{2}^{*}=7 / 15$. Further,

$$
\begin{aligned}
& {\left[\frac{L_{1} T^{\alpha}}{\Gamma(\alpha+1)}\left(1+\Omega_{1}^{*}\right)\left(1+p\left(1+L_{2}\right)+2 p \alpha+L_{3}\right)+\frac{L_{1} T^{\alpha-1}}{\Gamma(\alpha)} \Omega_{2}^{*}(1+p)+\left(\Omega_{1}^{*} T+\Omega_{2}^{*}+T\right) p L_{3}\right]} \\
& \quad=\frac{464}{1125 \sqrt{\pi}}+\frac{173}{450}<1 .
\end{aligned}
$$

Since the assumptions of Theorem 3.2 are satisfied, the closed boundary value problem (4.1) has a unique solution on $[0,1]$. Moreover, it is easy to check the conclusion of Theorem 3.3.

\section{Acknowledgments}

The authors would like to express their sincere thanks and gratitude to the reviewer(s) for their valuable comments and suggestions for the improvement of this paper. The second author also gratefully acknowledges that this research was partially supported by the University Putra Malaysia under the Research University Grant Scheme 05-01-09-0720RU.

\section{References}

[1] K. Diethelm, The Analysis of Fractional Differential Equations, Springer, 2010.

[2] N. Heymans and I. Podlubny, "Physical interpretation of initial conditions for fractional differential equationswith Riemann Liouville fractional derivatives," Rheologica Acta, vol. 45, no. 5, pp. 765-772, 2006.

[3] R. Hilfer, Applications of Fractional Calculus in Physics, World Scientific, Singapore, 2000.

[4] A. A. Kilbas, H. M. Srivastava, and J. J. Trujillo, Theory and Applications of Fractional Differential Equations, vol. 204 of North-Holland Mathematics Studies, Elsevier Science B.V., Amsterdam, The Netherlands, 2006.

[5] V. Lakshmikantham, S. Leela, and J. Vasundhara Devi, Theory of Fractional Dynamic Systems, Cambridge Academic Publishers, Cambridge, UK, 2009.

[6] J. Sabatier, O. P. Agrawal, and J. A. T. Machado, Eds., Advances in Fractional Calculus: Theoretical Developmentsand Applications in Physics and Engineering, Springer, Dordrecht, The Netherlands, 2007.

[7] S. G. Samko, A. A. Kilbas, and O. I. Marichev, Fractional Integrals and Derivatives. Theory and Applications, Gordon and Breach Science, Yverdon, Switzerland, 1993.

[8] R. P. Agarwal, M. Benchohra, and S. Hamani, "Boundary value problems for fractional differential equations," Georgian Mathematical Journal, vol. 16, no. 3, pp. 401-411, 2009.

[9] R. P. Agarwal, M. Benchohra, and S. Hamani, "A survey on existence results for boundary value problems of nonlinear fractional differential equations and inclusions," Acta Applicandae Mathematicae, vol. 109, no. 3, pp. 973-1033, 2010.

[10] Z. Bai and H. Lü, "Positive solutions for boundary value problem of nonlinear fractional differential equation," Journal of Mathematical Analysis and Applications, vol. 311, no. 2, pp. 495-505, 2005.

[11] M. Belmekki, J. J. Nieto, and R. Rodríguez-López, "Existence of periodic solution for a nonlinear fractional differential equation," Boundary Value Problems, vol. 2009, Article ID 324561, 18 pages, 2009.

[12] M. Benchohra, S. Hamani, and S. K. Ntouyas, "Boundary value problems for differential equations with fractional order and nonlocal conditions," Nonlinear Analysis, vol. 71, no. 7-8, pp. 2391-2396, 2009.

[13] H. A. H. Salem, "On the fractional order $m$-point boundary value problem in reflexive Banach spaces and weak topologies," Journal of Computational and Applied Mathematics, vol. 224, no. 2, pp. 565-572, 2009.

[14] W. Zhong and W. Lin, "Nonlocal and multiple-point boundary value problem for fractional differential equations," Computers \& Mathematics with Applications, vol. 59, no. 3, pp. 1345-1351, 2010. 
[15] V. Lakshmikantham, D. D. Baĭnov, and P. S. Simeonov, Theory of Impulsive Differential Equations, World Scientific, Singapore, 1989.

[16] Y. V. Rogovchenko, "Impulsive evolution systems: main results and new trends," Dynamics of Continuous, Discrete and Impulsive Systems, vol. 3, no. 1, pp. 57-88, 1997.

[17] A. M. Samorlenko and N. A. Perestyuk, Impulsive Differential Equations, World Scientific, Singapore, 1995.

[18] B. Ahmad and S. Sivasundaram, "Existence of solutions for impulsive integral boundary value problems of fractional order," Nonlinear Analysis, vol. 4, no. 1, pp. 134-141, 2010.

[19] M. Benchohra and B. A. Slimani, "Existence and uniqueness of solutions to impulsive fractional differential equations," Electronic Journal of Differential Equations, vol. 10, pp. 1-11, 2009.

[20] G. Wang, B. Ahmad, and L. Zhang, "Some existence results for impulsive nonlinear fractional differential equations with mixed boundary conditions," Computers \& Mathematics with Applications, vol. 62, no. 3, pp. 1389-1397, 2011.

[21] T. A. Burton and C. Kirk, "A fixed point theorem of Krasnoselskii-Schaefer type," Mathematische Nachrichten, vol. 189, pp. 23-31, 1998.

[22] A. Granas and J. Dugundji, Fixed Point Theory, Springer Monographs in Mathematics, Springer, New York, NY, USA, 2003. 


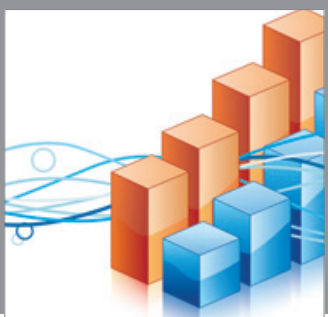

Advances in

Operations Research

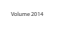

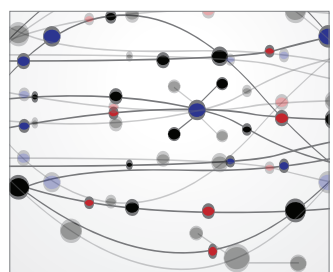

\section{The Scientific} World Journal
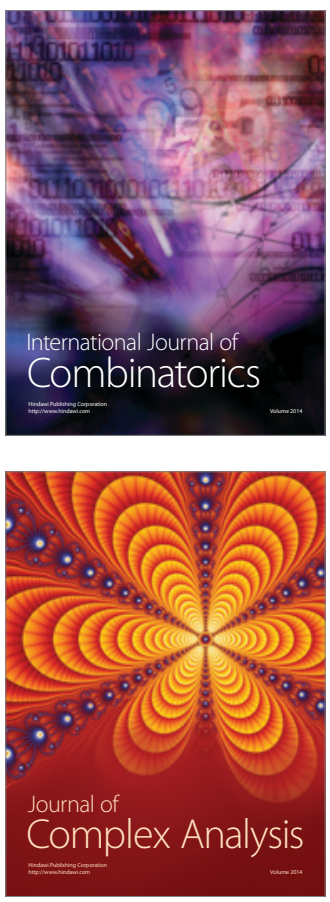

International Journal of

Mathematics and

Mathematical

Sciences
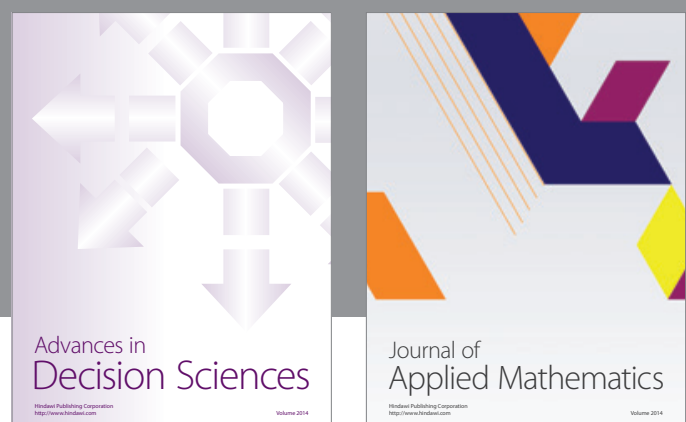

Journal of

Applied Mathematics
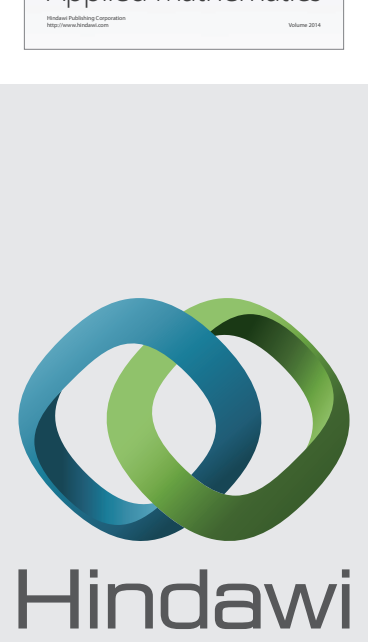

Submit your manuscripts at http://www.hindawi.com
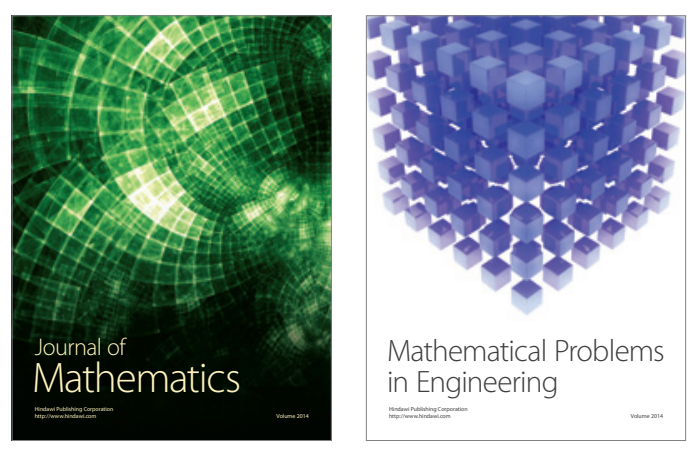

Mathematical Problems in Engineering
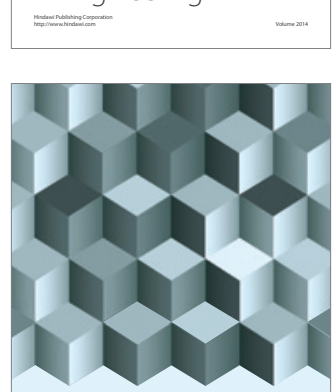

Journal of

Function Spaces
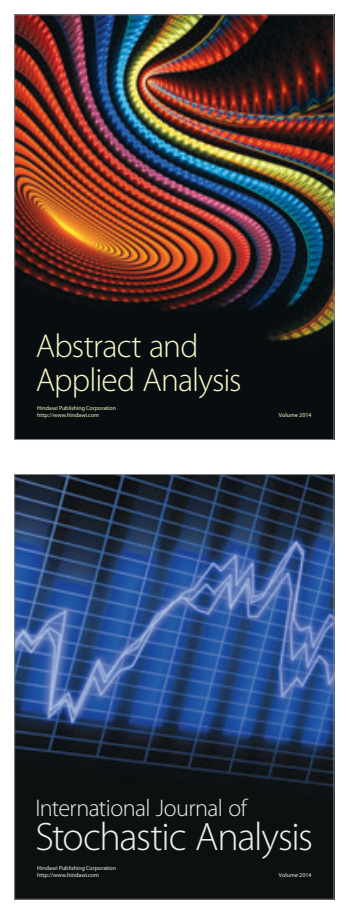

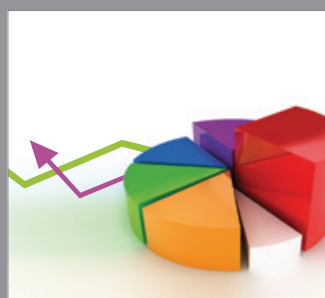

ournal of

Probability and Statistics

Promensencen
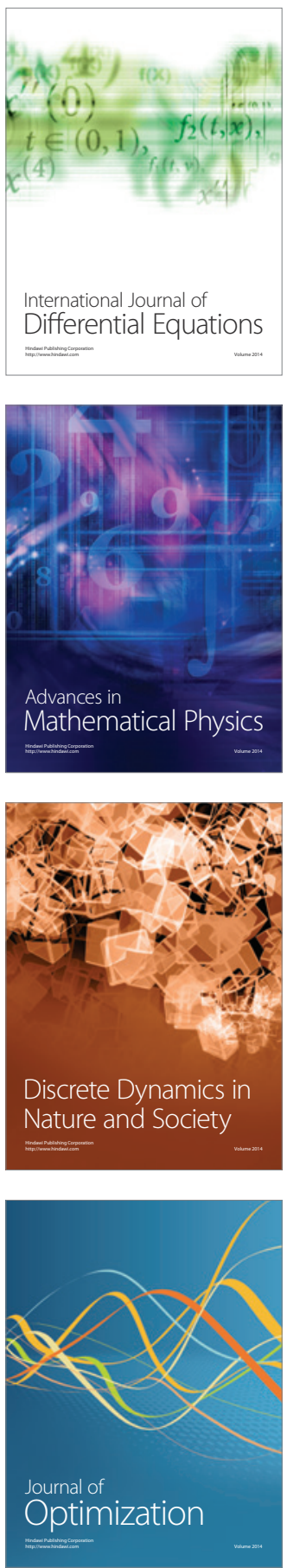